\title{
Fourier-Mukai partners of Enriques and bielliptic surfaces in positive characteristic
}

\author{
Katrina Honigs ${ }^{1}$, Max Lieblich ${ }^{2}$, and Sofia Tirabassi ${ }^{3,4}$ \\ ${ }^{1}$ University of Utah \\ ${ }^{2}$ University of Washington \\ ${ }^{3}$ University of Bergen \\ ${ }^{4}$ Stockholm University
}

\section{Contents}

1 Introduction 1

2 Twisted sheaves $\quad 2$

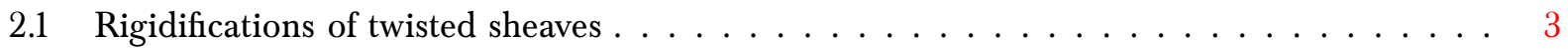

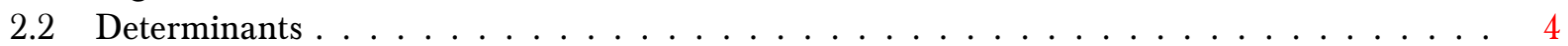

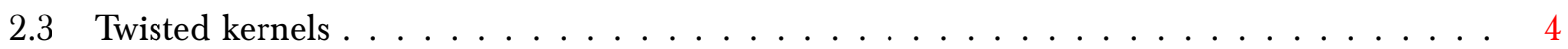

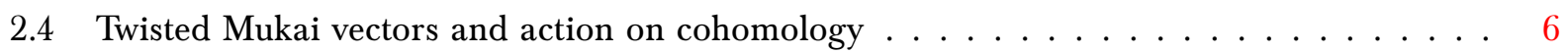

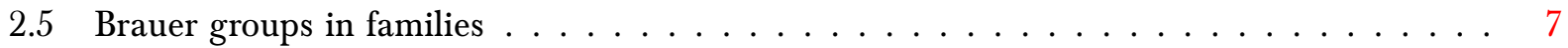

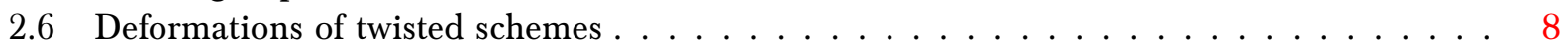

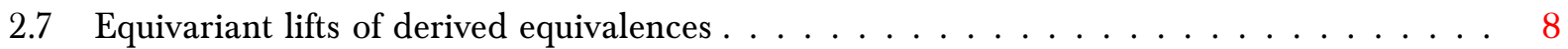

3 Deformations of twisted Fourier-Mukai kernels 10

3.1 Some results on the deformation theory of complexes $\ldots \ldots \ldots \ldots \ldots$

3.2 Moduli of perfect complexes of twisted sheaves . . . . . . . . . . . . . . . . 13

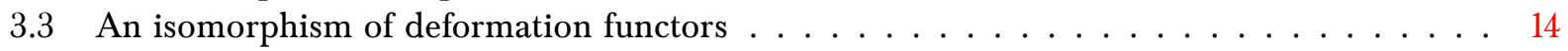

4 Proof of Theorem 1.1 and Theorem $1.2 \quad 16$

\section{Introduction}

A Fourier-Mukai partner of a smooth projective variety $X$ over a field $k$ is a smooth projective variety $Y$ over $k$ such that there is a $k$-linear equivalence of triangulated categories $D(X) \rightarrow D(Y)$, where $D(-)$ denotes the bounded derived category with coherent cohomology (equivalently - since $X$ and $Y$ are smooth - perfect complexes).

A twisted variety is a pair $(X, \alpha)$ with $X$ a variety and $\alpha \in \operatorname{Br}(X)$ a Brauer class. An isomorphism of twisted varieties $(X, \alpha) \rightarrow(Y, \beta)$ is an isomorphism $f: X \rightarrow Y$ such that $f^{*} \beta=\alpha$. Given a smooth projective twisted variety $(X, \alpha)$, we will let $D(X, \alpha)$ denote the bounded derived category of $\alpha$-twisted 
$\mathcal{O}_{X}$-modules with coherent cohomology. ${ }^{1}$ Another pair $(Y, \beta)$ is a twisted Fourier-Mukai partner of $(X, \alpha)$ if there is a $k$-linear equivalence of triangulated categories $D(X, \alpha) \rightarrow D(Y, \beta)$. We will say that $(X, \alpha)$ has no non-trivial twisted Fourier-Mukai partners if any twisted Fourier-Mukai partner partner $(Y, \beta)$ of $(X, \alpha)$ is isomorphic to $(X, \alpha)$.

In this note we prove the following results.

Theorem 1.1. If $(X, \alpha)$ is a twisted Enriques surface over an algebraically closed field $k$ of characteristic at least 3, then it does not have any nontrivial twisted Fourier-Mukai partners.

Theorem 1.2. If $X$ is a bielliptic surface over an algebraically closed field $k$ of characteristic at least 5 , then it does not have any nontrivial (untwisted) Fourier-Mukai partners.

These results were proven in the untwisted case over the complex numbers by Bridgeland and Maciocia [6]. Their argument uses Torelli theorems to reduce the result to lattice-theoretic assertions, and thus relies heavily on the complex numbers. The twisted cases of these results are proven over the complex numbers by Addington and Wray with methods analogous to those of Bridgeland and Maciocia, with the requisite modifications to handle the twists.

Our approach to proving this in positive characteristic is to lift (twisted) derived equivalences to characteristic 0 and specialize isomorphisms using the Matsusaka-Mumford theorem. The strategy is similar to that taken in [26]. In the present situation, the central issue is the question of whether the fibers of the kernel, viewed as complexes on $Y$, are smooth points in the moduli space of complexes, something that is automatic for K3 surfaces since obstruction spaces themselves vanish. While the obstruction spaces for simple complexes on Enriques and bielliptic surfaces are nonvanishing, we can nevertheless show that the obstruction classes vanish by using equivariant techniques similar to those originally used in [6].

In Section 2.7 we briefly discuss the positive characteristic version of the equivariance results of [5]. In Section 3 we work through producing an isomorphism of deformation spaces that gives us a way to lift kernels. The extension of the ideas of [23] to the twisted setting, discussed in Section 3.2, provides a framework for the argument. In Section 4 we complete the proof of Theorems 1.1 and 1.2.

\section{Acknowledgments}

Honigs was partially supported by NSF MSPRF grant DMS-160628 and thanks Nick Addington and Karl Schwede for helpful conversations. Lieblich was partially supported by NSF CAREER DMS-1056129, NSF standard grant DMS-1600813, and a Simons Foundation Fellowship; he thanks Martin Olsson for helpful remarks. Tirabassi was partially supported by grant 261756 of the Research Councils of Norway; she is grateful to Christian Liedtke and Richard Thomas for very interesting and stimulating mathematical discussions.

\section{Twisted sheaves}

In this section we develop enough of the theory of twisted schemes and moduli of perfect complexes of twisted sheaves to put our questions in families. This is well-trodden in the literature (see e.g. [9, 24, 39] for twisted sheaves and [23] for moduli of perfect complexes), so we only indicate the essentials here. The main distinction between our treatment here and the existing literature is our careful attention to the

\footnotetext{
${ }^{1}$ There are some subtle foundational issues with this definition as it is written. See Section 2.1 for a discussion of ways in which it can be corrected.
} 
distinction between $\mathbf{G}_{m}$-twisting and $\boldsymbol{\mu}_{n}$-twisting. We will occasionally have to pass between them, but we try to explain how the equivalences among the various models transfer.

\subsection{Rigidifications of twisted sheaves}

Definition 2.1.1. 1. A twisted scheme is a pair $(X, \alpha)$ with $X$ a scheme and $\alpha \in \operatorname{Br}(X)$.

2. A morphism of twisted schemes $(X, \alpha) \rightarrow(Y, \beta)$ is a morphism $f: X \rightarrow Y$.

3. The relative twisting class of a morphism $f:(X, \alpha) \rightarrow(Y, \beta)$ is the class $f^{*} \beta-\alpha$.

4. A morphism of twisted schemes is untwisted if the relative twisting class is 0 .

5. An isomorphism of twisted schemes is an untwisted morphism of twisted schemes whose underlying morphism $f: X \rightarrow Y$ is a isomorphism.

We will say that a morphism $f:(X, \alpha) \rightarrow(Y, \beta)$ has a property $P$ (e.g., is flat, proper, locally of finite presentation, etc.) if $P$ holds for the underlying morphism $f: X \rightarrow Y$.

Our purpose in defining twisted schemes is to study twisted sheaves. In order to properly handle the theory of determinants and Mukai vectors for different types of twisting coefficients $\left(\mathbf{G}_{m}\right.$ and $\left.\boldsymbol{\mu}_{n}\right)$, we will need to keep track of several categories at once.

Fix a twisted scheme $(X, \alpha)$ and an integer $n$ invertible in the base. Categories of $(n$-)twisted sheaves are not naturally associated to the pair $(X, \alpha)$, but rather to a "rigidification" of $\alpha$. There are several ways to choose this rigidification (see [25, §3.1] for more detail), which all yield equivalent categories of sheaves and, hence, derived categories of sheaves:

1. One type of rigidification uses a choice of Čech 2-cocycle representatives for $\alpha$ [9, Definition 1.2.1] and common in the complex-analytic literature, but we will not use it here.

2. Choose an Azumaya algebra $\mathscr{A}$ on $X$ representing $\alpha$. An $n$-fold twisted sheaf can then be identified with an $\mathscr{A}^{\otimes n}$-module.

3. Choose a closed subgroup $\mathbf{G} \subset \mathbf{G}_{m}$ and $\mathbf{G}$-gerbe $\mathscr{X} \rightarrow X$ whose associated Brauer class is $\alpha$. A locally free $\mathcal{O}_{\mathscr{X}}$-module $F$ has a natural sum decomposition by the weights of the right inertial action of $\mathbf{G}$ (cf. [30, §12.3])

$$
F \simeq \bigoplus_{i \in \mathbb{Z}} F_{i}
$$

where we index the characters $\mathbf{G} \rightarrow \mathbf{G}_{m}$ via the surjection $\mathbf{Z} \simeq \operatorname{Hom}\left(\mathbf{G}_{m}, \mathbf{G}_{m}\right) \rightarrow \operatorname{Hom}\left(\mathbf{G}, \mathbf{G}_{m}\right)$ : the $i$-th component has action given by scalar multiplication $i$ times. An $n$-fold twisted sheaf is a locally free $\mathscr{O}_{\mathscr{X}}$-module whose decomposition rests purely in weight $n$.

Remark 2.1.3. In the second model, the notions of flatness, quasi-coherence, coherence, homological dimension, etc., are all equivalent to the same notions for the underlying $\mathcal{O}_{X}$-module (since any Azumaya algebra is locally Morita-equivalent to the structure sheaf). In the third model, those notions are the usual ones for sheaves on a stack, and so we may seamlessly consider perfect complexes in either setting.

Unfortunately, the second realization does not preserve rank, determinant, etc, but the third is useful for studying determinants, ranks, Chern characters, etc., and so we work with this realization. 
Notation 2.1.4. We will write $\operatorname{Coh}^{(n)}(X, \alpha)$, resp. $\mathrm{QCoh}^{(n)}(X, \alpha)$, resp. $D^{(n)}(X, \alpha)$ for the category of coherent $n$-fold twisted sheaves, resp. quasi-coherent $n$-fold twisted sheaves, resp. bounded derived category of $n$-fold twisted sheaves with coherent cohomology. When we have a gerbe model $\mathscr{X} \rightarrow X$ of $\alpha$, we will write $\mathrm{QCoh}^{(n)}(\mathscr{X})$, etc., for the various categories of twisted sheaves.

\subsection{Determinants}

In this section we discuss the determinant of a perfect complex of twisted sheaves, using the gerbe definition of twisted sheaves. For some closed subgroup $\mathbf{G} \subset \mathbf{G}_{m}$, with dual group $\widehat{\mathbf{G}}:=\operatorname{Hom}\left(\mathbf{G}, \mathbf{G}_{m}\right)$, fix a $\mathrm{G}$-gerbe $\mathscr{X} \rightarrow X$. Let $\operatorname{Pic}(\mathscr{X})$ be the group of invertible sheaves on $\mathscr{X}$. The decomposition (2.1.2) shows the elements of $\operatorname{Pic}(\mathscr{X})$ must have pure weight and hence there is a natural map taking a sheaf to its character:

$$
\operatorname{Pic}(\mathscr{X}) \rightarrow \widehat{\mathbf{G}}
$$

Since the group law on $\widehat{\mathbf{G}}$ is determined by tensoring characters together, this morphism is a group homomorphism.

Lemma 2.2.1. Given a perfect complex $P \in D^{(a)}(\mathcal{Y})$ of rank $r$, the determinant sheaf $\operatorname{det}(P)$ lies in $\operatorname{Pic}^{(r a)}(\mathscr{Y}) \subset \operatorname{Pic}(\mathscr{Y})$.

Proof. It suffices to prove this after passing to a geometric fiber of $\mathcal{Y} \rightarrow Y$, so we may assume that $\mathscr{Y}=\mathbf{B G}_{K}$ for some algebraically closed field $K$. A perfect complex of $a$-fold twisted sheaves of rank $r$ is then identified with a graded G-representation that has graded rank $r$ and whose underlying Grepresentation is isotypic of type $a$. Since the determinant is computed as the alternating tensor product of the wedge powers of the factors, we see that the type of the determinant is precisely $r a$, as desired.

\subsection{Twisted kernels}

By [8], a large class of exact functors $D(X, \alpha) \rightarrow D(Y, \beta)$, including equivalences, are given by FourierMukai transforms. In the classical literature on twisted Fourier-Mukai functors, the kernel is given as an object in $D\left(X \times Y, \alpha^{-1} \otimes \beta\right)$, where $\otimes$ denotes the tensor product of the pullbacks of $\alpha^{-1}$ and $\beta$ to the product $X \times Y$.

However, it is a priori unclear how, in the gerbe point of view, Fourier-Mukai functors constructed using the objects in $D\left(X \times Y, \alpha^{-1} \otimes \beta\right)$, which are 1-twisted perfect complexes on a G-gerbe, yield a map from 1-twisted sheaves on a gerbe associated to $\alpha$ to 1 -twisted sheaves on a gerbe associated to $\beta$. There is an identification hiding in the background of many treatments of this subject that makes our gerbe approach to twisted Fourier-Mukai kernels equivalent to the above definition, which we take the opportunity to discuss here.

Fix a closed subgroup $\mathbf{G} \subset \mathbf{G}_{m}$. Suppose $Y$ is a quasi-compact quasi-separated scheme and $\mathscr{Z} \rightarrow Y$ and $\mathscr{Z}^{\prime} \rightarrow Y$ are $\mathbf{G}$-gerbes. Given a quasi-coherent sheaf $F$ on $\mathscr{Z} \times_{Y} \mathscr{Z}^{\prime}$, we may use the weights of the G-action on $p_{\mathscr{X}}^{*} F$ and $p_{\mathscr{Z}^{\prime}}^{*} F$ to decompose $F$ as follows:

$$
F=\bigoplus_{\widehat{\mathbf{G}} \times \widehat{\mathbf{G}}} F_{(i, j)}
$$

Given $i, j \in \widehat{\mathbf{G}}$ we write $\mathrm{QCoh}^{(i, j)}\left(\mathscr{Z} \times \mathscr{Z}^{\prime}\right)$ for the subcategory of quasi-coherent sheaves $F$ such that $F=F_{(i, j)}$. 
Since any morphism $\mathbf{G} \rightarrow \mathbf{G}_{m}$ factors uniquely through $\mathbf{G}$, we may view $\widehat{\mathbf{G}}$ as $\operatorname{Hom}(\mathbf{G}, \mathbf{G})$. Given a pair $(i, j) \in \widehat{\mathbf{G}} \times \widehat{\mathbf{G}}$, define a map

$$
\sigma_{(i, j)}: \mathbf{G} \times \mathbf{G} \rightarrow \mathbf{G}
$$

by sending $(a, b)$ to $i(a) \cdot j(b)$. There is an induced map

$$
\left(\sigma_{(i, j)}\right)_{*}: \mathrm{H}^{2}(Y, \mathbf{G} \times \mathbf{G}) \rightarrow \mathrm{H}^{2}(Y, \mathbf{G})
$$

that we can realize on the level of stacks (using Giraud's theory [13]) as follows: given a pair of G-gerbes $\mathscr{Z} \rightarrow Y$ and $\mathscr{Z}^{\prime} \rightarrow Y$, there is a $\mathbf{G}$-gerbe $\left(\mathscr{Z} \wedge_{(i, j)} \mathscr{Z}^{\prime}\right)$ and a morphism

$$
\tau\left(\mathscr{Z}, \mathscr{Z}^{\prime}, i, j\right): \mathscr{Z} \times_{Y} \mathscr{Z}^{\prime} \rightarrow\left(\mathscr{Z} \wedge_{(i, j)} \mathscr{Z}^{\prime}\right)
$$

of $Y$-stacks whose induced morphism on inertia is precisely $\sigma_{(i, j)}$. The morphism is locally modeled on the composition

$$
\mathrm{BG} \times \mathrm{BG} \rightarrow \mathrm{B}(\mathrm{G} \times \mathrm{G}) \rightarrow \mathrm{BG},
$$

where the first map is the canonical one and the second is induced by $\sigma_{(i, j)}$.

Lemma 2.3.1. Let $\mathscr{Z}$ and $\mathscr{Z}^{\prime}$ be $\mathbf{G}$-gerbes on $Y$ and $\sigma_{(i, j)}: \mathbf{G} \times \mathbf{G} \rightarrow \mathbf{G}$ a morphism as above. Pulling back by $\tau\left(\mathscr{Z}, \mathscr{Z}^{\prime}, i, j\right)$ induces an equivalence of categories

$$
\mathrm{QCoh}^{(1)}\left(\mathscr{Z} \wedge_{(i, j)} \mathscr{Z}^{\prime}\right) \rightarrow \mathrm{QCoh}^{(i, j)}\left(\mathscr{Z} \times \mathscr{Z}^{\prime}\right)
$$

Proof. These categories are both the global sections of stacks on $Y$, so to prove the statement it suffices to assume that $\mathscr{Z}$ and $\mathscr{Z}^{\prime}$ are both isomorphic to $\mathbf{B G}$, in which case our morphism becomes $\mathbf{B} \sigma_{(i, j)}$. The statement then follows since the $(i, j)$-eigensheaf is precisely the sheaf with action factoring through $\sigma_{(i, j)}$.

Since $X$ and $X^{\prime}$ are quasi-compact and quasi-separated, we have that the bounded derived category of $\mathcal{O}$-modules with quasi-coherent cohomology is equivalent to the bounded derived category of quasicoherent sheaves. Thus we have the following corollary:

Corollary 2.3.2. With the above notation, we have that $\sigma_{(i, j)}^{*}$ identifies the derived category of perfect complexes of $\mathscr{Z} \wedge_{(i, j)} \mathscr{Z}^{\prime}$-twisted sheaves with the derived category of perfect complexes on $\mathscr{Z} \times_{Y} \mathscr{X}^{\prime}$ of type $(i, j)$.

Definition 2.3.3. Given two G-gerbes $X \rightarrow X$ and $\mathscr{Y} \rightarrow Y$, a twisted Fourier-Mukai kernel is a perfect complex $P \in D^{(-1,1)}(\mathscr{X} \times \mathscr{Y})$. The Fourier-Mukai transform associated to $P$ is the functor

$$
\Phi_{P}: D^{(1)}(\mathscr{X}) \rightarrow D^{(1)}(\mathscr{Y})
$$

defined by

$$
\Phi_{P}(a)=\mathbf{R}\left(\operatorname{pr}_{2}\right)_{*}\left(\mathbf{L} \operatorname{pr}_{1}^{*} a \stackrel{\mathbf{L}}{\otimes} P\right)
$$

By Corollary 2.3.2, this definition is equivalent to the one discussed at the beginning of the section.

These Fourier-Mukai equivalences commute with Serre functors, hence we have the following result:

Lemma 2.3.4. If there is a Fourier-Mukai equivalence $\Phi_{P}: D^{(1)}(X, \alpha) \rightarrow D^{(1)}(Y, \beta)$ then $\operatorname{dim} X=\operatorname{dim} Y$ and $\left[\omega_{X}\right] \in \operatorname{Pic}(X)$ and $\left[\omega_{Y}\right] \in \operatorname{Pic}(Y)$ have the same order. 


\subsection{Twisted Mukai vectors and action on cohomology}

In this section we briefly consider the theory of twisted Mukai vectors, which will be essential for showing the vanishing of certain obstructions in Section 3.1 below.

Let $X$ be a smooth projective variety over a field $k$ and $\pi: \mathscr{X} \rightarrow X$ be a $\boldsymbol{\mu}_{n}$-gerbe for some $n$ invertible in $k$. Since $\pi$ is the coarse moduli map of an Deligne-Mumford stack, there is an induced isomorphism on Chow theory (with respect to rational equivalence) with rational coefficients [12, Theorem 0.5]:

$$
\pi_{*}: A^{*}(\mathscr{X})_{\mathbf{Q}} \rightarrow A^{*}(X)_{\mathbf{Q}}
$$

We can use this to define a Chern character and Mukai vector for any perfect complex of $\mathscr{X}$-twisted sheaves, as in [4, §4.1].

Definition 2.4.1. In this setting, $\mathscr{X} \rightarrow X$ has the resolution property, so we may define the Chern character of a perfect complex of $\mathscr{X}$-twisted sheaves by extending the following:

1. Given a locally free $\mathscr{X}$-twisted sheaf $\mathscr{E}$, the Chern character of $P$ is the class

$$
\operatorname{ch}(\mathscr{E}):=\operatorname{ch}\left(\mathscr{E}^{\otimes n}\right)^{1 / n} \in A^{*}(X)_{\mathbf{Q}}
$$

chosen so that its component in $A^{0}(X)_{\mathrm{Q}}$ has the same sign as the rank of $\mathscr{E}$;

2. The Mukai vector of $P$ is the class

$$
v(P):=\operatorname{ch}(P) \cdot \sqrt{\mathrm{Td}_{X}} \in A^{*}(X)_{\mathbf{Q}} .
$$

Remark 2.4.2. This definition agrees over $\mathbf{C}$ with the twisted Chern character defined by Huybrechts in $[18, \S 2.1]$. It differs by a factor from the definition introduced in [19] (see [18, §1.3] for a comparison), which is more noncanonical as it depends on a choice of $B$-field, but has the advantage of integrality.

Now suppose that $X \rightarrow X$ and $\mathscr{Y} \rightarrow Y$ are $\boldsymbol{\mu}_{n}$-gerbes over smooth projective varieties, and let $P \in D^{(-1,1)}(\mathscr{X} \times \mathscr{Y})$ be a twisted Fourier-Mukai kernel. Its Mukai vector can be used to define the following map:

$$
\begin{aligned}
& \Psi_{v(P)}: A^{*}(X)_{\mathbf{Q}} \rightarrow A^{*}(Y)_{\mathbf{Q}} \\
& \Psi_{v(P)}(x)=\left(\operatorname{pr}_{2}\right)_{*}\left(\operatorname{pr}_{1}^{*} x \cup v(P)\right) .
\end{aligned}
$$

The following is a well-known compatibility between the various transforms.

Lemma 2.4.3. For any $a \in D^{(1)}(\mathscr{X})$ we have

$$
\Psi_{v(P)}(v(a))=v\left(\Phi_{P}(a)\right)
$$

as elements of $A^{*}(Y) \mathbf{Q}$.

Proof. The proof in the untwisted case is a simple application of the Grothendieck-Riemann-Roch formula. The twisted case is a bit more subtle; a proof can be found in [37] (cf., for instance, [7, Appendix A]).

Corollary 2.4.4. Suppose $\omega_{Y}$ is torsion. If $a, b \in D^{(1)}(\mathscr{X})$ satisfy $v(a)=v(b)$, then

$$
\operatorname{det}\left(\Phi_{P}(a)\right)=\operatorname{det}\left(\Phi_{P}(b)\right) \in \operatorname{Pic}(\mathscr{Y}) \otimes \mathbf{Q}=\operatorname{Pic}(Y) \otimes \mathbf{Q} .
$$


Proof. From the formula for the Todd class, we have that

$$
\operatorname{Td}_{Y}=1-\frac{1}{2} c_{1}\left(\omega_{Y}\right)+\cdots \quad \text { and hence } \quad \sqrt{\mathrm{Td}_{Y}}=1-\frac{1}{4} c_{1}\left(\omega_{Y}\right)+\cdots .
$$

It follows that the component of $v\left(\Phi_{P}(a)\right)$ in $A^{1}(Y)_{\mathbf{Q}}$ is

$$
v_{1}\left(\Phi_{P}(a)\right)=\operatorname{det}\left(\Phi_{P}(a)\right)-\frac{1}{4} \operatorname{rk}\left(\Phi_{P}(a)\right) c_{1}\left(\omega_{Y}\right) .
$$

The result now follows from the fact that $\omega_{Y}$ is torsion, so that $c_{1}\left(\omega_{Y}\right)=0 \in A^{*}(Y)_{\mathbf{Q}}$.

Applying the usual cycle class maps to $v(P)$ also defines induced correspondences on rational cohomology theories. (See [26, §2] for more information.) In particular, we have the following.

Lemma 2.4.5. Suppose $H$ is a Weil cohomology theory and $(X, \alpha)$ and $(Y, \beta)$ are two smooth projective twisted varieties. Any derived equivalence

$$
\Phi_{P}: D^{(1)}(X, \alpha) \rightarrow D^{(1)}(Y, \beta)
$$

induces isomorphisms

$$
\Phi_{P}^{\text {odd }}: H^{\text {odd }}(X) \rightarrow H^{\text {odd }}(Y) \quad \text { and } \quad \Phi_{P}^{\text {even }}: H^{\text {even }}(X) \rightarrow H^{\text {even }}(Y) .
$$

Proof. See [16, Lemma 3.1] for details.

\subsection{Brauer groups in families}

In this section we briefly discuss the variation of the Brauer group in families of Enriques and bielliptic surfaces. We recall that, for a scheme $X$, the Brauer group of $X, \operatorname{Br}(X)$ is the group of Azumaya algebras over $X$ up to Morita equivalence. The cohomological Brauer group of $X$ is $\mathrm{H}_{\text {êt }}^{2}\left(X, \mathbf{G}_{m}\right)_{\text {tors. }}$. There is a canonical inclusion $\operatorname{Br}(X) \hookrightarrow \mathrm{H}_{\text {êt }}^{2}\left(X, \mathbf{G}_{m}\right)_{\text {tors }}$, which is an isomorphism whenever $X$ is quasi-compact and separated. The reader is referred to [15] and [10] for details. Under these assumptions we will denote both the Brauer group and the cohomological Brauer group by $\operatorname{Br}(X)$.

Suppose $\pi: X \rightarrow S$ is a proper morphism that is cohomologically flat in dimension 0 (see [14, 6.1.2]). Fix a positive integer $n$ that is invertible on $S$. We assume that the Brauer group and cohomological Brauer group coincide for each geometric fiber of $\pi$; this holds, for example, if $\pi$ is locally projective or if the fibers are smooth of dimension 2.

Let $\overline{\mathrm{Br}}_{X / S}$ be the sheaf associated to the presheaf that sends $T \rightarrow S$ to the Brauer group $\operatorname{Br}\left(X_{T}\right)$. There is a natural injective map of sheaves

$$
\overline{\mathrm{Br}}_{X / S} \rightarrow \mathbf{R}^{2} \pi_{*} \mathbf{G}_{m}
$$

on the big étale site of $S$. This induces an injection

$$
\overline{\mathrm{Br}}_{X / S}[n] \rightarrow \mathbf{R}^{2} \pi_{*} \mathbf{G}_{m}[n]
$$

Proposition 2.5.1. Suppose that for all $T \rightarrow S$ we have $\mathbf{R}^{2}\left(\pi_{T}\right)_{*} \mathcal{O}_{X_{T}}=0$. Then the sheaf $\overline{\mathrm{Br}}_{X / S}[n]$ is represented by a finite étale cover of $S$. In particular, if $S$ is strictly Henselian then restriction defines isomorphisms

$$
\operatorname{Br}\left(X_{K}\right)^{\prime} \leftarrow \operatorname{Br}(X)^{\prime} \rightarrow \operatorname{Br}\left(X_{k}\right)^{\prime}
$$

where $k$ is the residue field at the closed point of $S, K$ is an algebraic closure of the function field of $S$, and $\operatorname{Br}()^{\prime}$ denotes the subgroup of the Brauer group consisting of classes with order prime to the characteristic exponent of $k$. 
Proof. Consider the short exact sequence

$$
0 \rightarrow \mathrm{Pic}_{X / S} \otimes \mathbf{Z} / n \mathbf{Z} \rightarrow \mathbf{R}^{2} \pi_{*} \boldsymbol{\mu}_{n} \rightarrow \mathbf{R}^{2} \pi_{*} \mathbf{G}_{m}[n] \rightarrow 0 .
$$

Since $\mathrm{H}^{2}\left(X_{s}, \mathcal{O}_{X_{s}}\right)$ vanishes in every fiber, we see that $\mathrm{Pic}_{X / S}$ formally smooth, whence $\mathrm{Pic}_{X / S} \otimes \mathbf{Z} / n \mathbf{Z}$ is locally constant (as it is an algebraic space that is finite and étale over the base). The proper and smooth base change theorems in étale cohomology tell us the same thing about $\mathbf{R}^{2} \pi_{*} \boldsymbol{\mu}_{n}$. It follows that the quotient

$$
Q:=\mathbf{R}^{2} \pi_{*} \boldsymbol{\mu}_{n} / \mathrm{Pic}_{X / S} \otimes \mathbf{Z} / n \mathbf{Z}
$$

is a locally constant sheaf of finite abelian groups.

By assumption, for each geometric fiber of $X / S$ the inclusion

$$
\overline{\mathrm{Br}}_{X / S}[n] \subset Q
$$

is surjective on each geometric fiber, hence is an isomorphism of sheaves. Applying this for all $n$ invertible in $S$ yields the desired result.

\subsection{Deformations of twisted schemes}

Definition 2.6.1. Given a flat twisted space $(X, \alpha)$ over a scheme $S$ and a closed immersion $S \rightarrow S^{\prime}$, a deformation of $(X, \alpha)$ over $S^{\prime}$ is a flat twisted space $\left(X^{\prime}, \alpha^{\prime}\right)$ over $S^{\prime}$ together with an isomorphism $X \rightarrow X_{S}^{\prime}$ such that $\alpha_{X}^{\prime}=\alpha$.

Given an algebraically closed field $k$, let $W(k)$ denote the Witt vectors of $k$.

Definition 2.6.2. The category $\operatorname{Art}_{W}^{k}$ is the category of Artinian augmented $W$-algebras: an object is a local Artinian $W$-algebra $W \rightarrow(A, \mathfrak{m})$ together with an identification $k \rightarrow A / \mathfrak{m}$.

Definition 2.6.3. Suppose $k$ is algebraically closed. Given a twisted $k$-variety $(X, \alpha)$, the deformation functor of $(X, \alpha)$ is the functor

$$
\operatorname{Def}_{(X, \alpha)}: \operatorname{Art}_{W}^{k} \rightarrow \text { Set }
$$

that sends $A$ to the set of isomorphism classes of deformations of $(X, \alpha)$ over $\operatorname{Spec} A$.

Corollary 2.6.4. If $X$ is a smooth projective surface overk such that $\mathrm{H}^{2}\left(X, \mathcal{O}_{X}\right)=0$ then for any $\alpha \in \operatorname{Br}(X)$ with order prime to the characteristic exponent of $k$ the forgetful morphism

$$
\operatorname{Def}_{(X, \alpha)} \rightarrow \operatorname{Def}_{X}
$$

is an isomorphism.

Proof. This follows from Proposition 2.5.1, since the sheaf of Brauer groups is finite étale, hence splits over strictly Henselian rings.

\subsection{Equivariant lifts of derived equivalences}

Fix a base scheme $S=\operatorname{Spec}(A)$ with $A$ a strictly Henselian local ring, and fix proper smooth twisted schemes $(X, \alpha) \rightarrow S$ and $(Y, \beta) \rightarrow S$. Suppose the relative dualizing sheaves $\omega_{X / S}$ and $\omega_{Y / S}$ are torsion of order $n$, with $n$ invertible on $S$. Given trivializations $\omega_{X / S}^{\otimes n} \stackrel{\sim}{\rightarrow} \mathcal{O}_{X}$ and $\omega_{Y / S}^{\otimes n} \stackrel{\sim}{\rightarrow} \mathcal{O}_{Y}$, let $\widetilde{X} \rightarrow X$ and $\widetilde{Y} \rightarrow Y$ be the finite étale covers corresponding to the associated classes in $\mathrm{H}^{1}\left(X, \boldsymbol{\mu}_{n}\right)$ and $\mathrm{H}^{1}\left(Y, \boldsymbol{\mu}_{n}\right)$, respectively (see [35, Tag 03PK] for a discussion of this correspondence). 
Bridgeland and Maciocia [5] studied the problem of lifting derived equivalences $D(X) \rightarrow D(Y)$ to $D(\widetilde{X}) \rightarrow D(\widetilde{Y})$ when $S=$ Spec $\mathbf{C}$ and the twisting classes are trivial. In [1, Proposition 2.1], the authors extend this work to lifting equivalences between derived categories of coherent twisted sheaves, also over C. The argument is given in the particular setting of Enriques surfaces, where $\omega_{X}^{\otimes 2} \cong \mathcal{O}_{X}$, but it translates immediately to the general case of smooth, projective varieties with torsion canonical bundle.

When $n$ is invertible in the base, the methods of $[5,1]$ can be adapted to prove a relative form of these results. With this assumption, the proofs translate word-for-word. However, since there are some subtleties in checking this that are discussed elsewhere but in different sources, we assemble a discussion or references for all of them for the reader's convenience.

Proposition 2.7.1. Let $\mathscr{X} \rightarrow X$ and $\mathscr{Y} \rightarrow Y$ be $\mu_{n}$-gerbes representing $\alpha$ and $\beta$, and we write $\widetilde{\mathscr{X}}:=\mathscr{X} \times_{X} \widetilde{X}$ and $\tilde{\mathcal{Y}}:=\mathscr{Y} \times_{Y} \widetilde{Y}$. Given a Fourier-Mukai equivalence

$$
\Phi_{P}: D^{(1)}(\mathscr{X}) \rightarrow D^{(1)}(\mathscr{Y})
$$

with kernel

$$
P \in D^{(-1,1)}\left(\mathscr{X} \times_{S} \mathscr{Y}\right)
$$

there exists

$$
\widetilde{P} \in D^{(-1,1)}\left(\tilde{X} \times_{S} \tilde{\mathscr{Y}}\right)
$$

so that

$$
\Phi_{\widetilde{P}}: D^{(1)}(\widetilde{\mathscr{X}}) \rightarrow D^{(1)}(\widetilde{\mathscr{Y}})
$$

is an equivalence and the following diagrams commute:
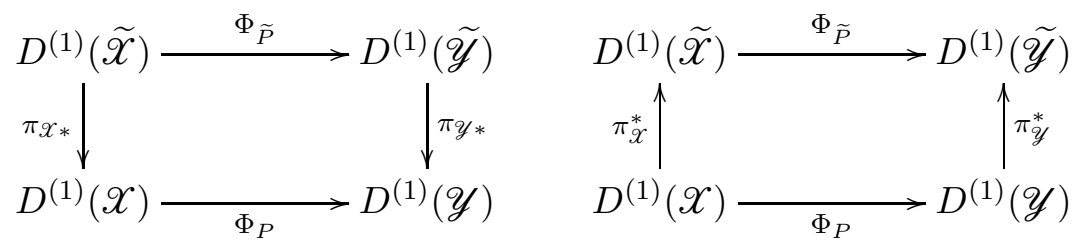

An equivalence $\Phi_{\widetilde{P}}$ making the diagrams (2.7.2) commute is called lift of $\Phi_{P}$.

Proof. The methods rely on the following result about equivariant objects for cyclic coverings. Suppose $Z$ is a Deligne-Mumford stack such that every element of $\Gamma\left(Z, \mathcal{O}_{Z}\right)$ is an $n$th power (for example, $Z$ is proper over $A$, since $A$ is strictly Henselian and $n$ is invertible on $A$ ), $L$ is an invertible sheaf on $Z$, and $\sigma: L^{\otimes n} \rightarrow \mathcal{O}_{Z}$ is an isomorphism. Write

$$
\widetilde{Z}=\underline{\operatorname{Spec}}_{Z} \bigoplus_{i=1}^{n-1} L^{\otimes i} \rightarrow Z
$$

for the $\boldsymbol{\mu}_{n}$-cover associated to the pair $(L, \sigma)$. Given a simple object $E$ of $D_{\mathrm{QCoh}}(Z)$ (the bounded derived category with quasi-coherent cohomology), there is some $\widetilde{E} \in D_{\mathrm{QCoh}}(\widetilde{Z})$ such that $p_{*} \widetilde{E} \cong E$ if and only if there is an isomorphism $\lambda: E \otimes L \rightarrow E$.

To prove this, we first proceed by induction and use exactness of tensoring with $L$ to reduce to the case that $E$ is a quasi-coherent sheaf as in [5, Proposition 2.5]. The isomorphism $\lambda$ induces an action of $\bigoplus_{i=0}^{n-1} L^{\otimes i}$ on $E$. It follows from the definition of (2.7.3) that sheaves with such an action that is furthermore compatible with $\sigma$ are naturally equivalent to pushforwards of $\mathcal{O}_{\widetilde{Z}}$-modules. 
There is a subtle point here: the action of $\bigoplus L^{\otimes i}$ on $E$ must be made compatible with the $\mathcal{O}_{Z^{-}}$ module action $\sigma$. Let $\varphi: E \otimes L^{\otimes n} \rightarrow E$ be the action of $L^{\otimes n}$ on $E$ induced by $\lambda$. Consider the composition $(\sigma \otimes E) \circ \varphi^{-1} \in \operatorname{Aut}(E)$. Since we have required $E$ to be simple, we have $\operatorname{Aut}(E)=\operatorname{End}(E)^{\times}=\mathscr{O}_{Z}(Z)^{\times}$, and because we have required that all elements of $\mathcal{O}_{Z}(Z)$ be $n$th roots, we can take an $n$th root of this composition, call it $\psi$. Replacing $\lambda$ with $\psi \circ \lambda$ yields an isomorphism with the needed compatibility.

The kernel $\widetilde{P}$ is constructed by applying this result to find a lift of $\left(\pi_{X} \times \operatorname{id}_{Y}\right)^{*} P$ to $\widetilde{X} \times \widetilde{Y}$; since $P$ is a kernel of a Fourier-Mukai equivalence, it is simple, so furthermore $\left(\pi_{X} \times \operatorname{id}_{Y}\right)^{*} P$ is simple; cf. [1, Proposition 2.1]. (Note that simplicity is omitted from [5, Proposition 2.5(b)].) By construction, we have that the diagram in the statement of the theorem commutes.

The argument given in, for instance, [17, Proposition 7.18] shows that the restriction of $\Phi_{\widetilde{P}}$ to the residue field of $A$ is an equivalence. To show that $\Phi_{\widetilde{P}}$ is an equivalence, we appeal to a derived form of the Nakayama lemma. That is: $\Phi_{\widetilde{P}}$ has left and right adjoints, with kernels $\widetilde{L}$ and $\widetilde{R}$. The adjunctions induce maps on kernels $\widetilde{L} \circ \widetilde{P} \rightarrow \Delta_{*} \mathcal{O}_{X}$ and $\Delta_{*} \mathcal{O}_{y} \rightarrow \widetilde{P} \circ \widetilde{R}$. The restrictions of these morphisms to the residue field of $A$ are isomorphisms by [5, Lemma 4.3(a)], whose proof works over any separably closed field in which $n$ is invertible. Hence, by Nakayama's Lemma, $\Phi_{\widetilde{P}}$ is an equivalence.

\section{Deformations of twisted Fourier-Mukai kernels}

\subsection{Some results on the deformation theory of complexes}

Let $A \rightarrow A_{0}$ a square-zero extension of rings with kernel $I$.

Lemma 3.1.1. Let $\gamma:(Z, \alpha) \rightarrow(X, \beta)$ be an untwisted finite étale morphism of flat separated twisted schemes over A. Let $(Z, \alpha)_{A_{0}}$ be the twisted scheme $\left(\left.Z\right|_{A_{0}},\left.\alpha\right|_{A_{0}}\right)$. Given a perfect complex $P \in D\left((Z, \alpha)_{A_{0}}\right)$, the natural map

$$
\operatorname{Ext}_{X_{0}}^{2}(P, P \otimes I) \rightarrow \operatorname{Ext}_{Z_{0}}^{2}\left(\mathbf{L} \gamma^{*} P, \mathbf{L} \gamma^{*} P \stackrel{\mathbf{L}}{\otimes} I\right)
$$

sends the obstruction to deforming $P$ over $A$ to the obstruction to deforming $\mathbf{L} \gamma^{*} P$ over $A$.

Proof. The proof of this result we offer here is undoubtedly far from ideal. Unfortunately, there does not appear to be an argument in the literature that is general enough to work without resorting to the techniques used in [23, Section 3]. The idea there is as follows: given the complex $P$, one replaces it by a "good resolution" $P^{\prime} \rightarrow P$, which is a quasi-isomorphism in which $P^{\prime}$ has terms of the form $\oplus j ! \mathcal{O}_{U}$ for étale morphisms $j: U \rightarrow X$ with $U$ affine. The complex $P^{\prime}$ is itself $K$-flat (in the sense of [34]), so it can be used to compute derived tensor products. The obstruction class arises by computing good resolutions of $P$ over $A_{0}$ and over $A$ and then making an explicit map

$$
Q \rightarrow P \stackrel{\mathbf{L}}{\otimes}_{A_{0}} I[1]
$$

in $D^{b}\left(X_{0}\right)$, where $Q$ is the homotopy limit of the derived adjunction map $P \stackrel{\mathbf{L}}{\otimes_{A}} A_{0} \rightarrow P$. (Details may be found in [23, Construction 3.2.8].) To adapt this to the twisted setting, we replace $\mathcal{O}$ with an Azumaya algebra $\mathscr{A}$ representing $\beta$. The rest of the arguments carry over verbatim.

Consider the Cartesian diagram

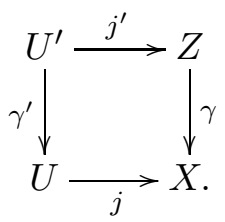


To establish the appropriate functoriality of the obstruction class, it suffices to show that $\gamma^{*} j_{!} \mathscr{A}_{U}=j_{!}^{\prime} \mathscr{A}_{U^{\prime}}$. By flat base change we have a canonical isomorphism of functors $j^{*} \gamma_{*}=\gamma_{*}^{\prime}\left(j^{\prime}\right)^{*}$, giving rise to a canonical isomorphism $\gamma^{*} j_{!}=j_{!}^{\prime}\left(\gamma^{\prime}\right)^{*}$. This gives the desired result.

In what follows, we will use a result that is essentially a folk theorem. We state it here as a conjecture; a proof will appear in [27].

Conjecture 3.1.3. Let $X \rightarrow \operatorname{Spec} A$ be a flat Artin stack such that $\omega_{X / A} \cong \mathscr{O}_{X}$ and let $X_{0}=X \otimes_{A} A_{0}$. Suppose $P_{0}$ is a perfect complex on $X_{0}$ with determinant $L_{0}$. Let

$$
o\left(P_{0}\right) \in \operatorname{Ext}_{X_{0}}^{2}\left(P_{0}, P_{0} \stackrel{\mathbf{L}}{\otimes} I\right)
$$

and

$$
o\left(L_{0}\right) \in \operatorname{Ext}_{X_{0}}^{2}(\mathcal{O}, \mathcal{O} \stackrel{\mathbf{L}}{\otimes} I)
$$

be the obstruction classes to the deformation of $P_{0}$ and $L_{0}$ to $X$. Then the trace map

$$
\operatorname{Ext}_{X_{0}}^{2}\left(P_{0}, P_{0} \stackrel{\mathbf{L}}{\otimes} I\right) \rightarrow \operatorname{Ext}_{X_{0}}^{2}(\mathcal{O}, \mathcal{O} \stackrel{\mathbf{L}}{\otimes} I)
$$

sends $o\left(P_{0}\right)$ to $o\left(L_{0}\right)$.

Remark 3.1.5. Note that Conjecture 3.1 .3 is phrased for a flat Artin stack, so it doesn't fit into the usual Illusie topos framework. Nevertheless, the deformation-obstruction theory for perfect complexes has the same formal propertiers as in the classical topos-theoretic case by the alternative argument of Grothendieck [20, IV.3.1.12].

Remarks on the state of Conjecture 3.1.3. As far as we can tell, there is no proof anywhere in the literature even in the case of deformations of a coherent sheaf. The proof for locally free sheaves on projective varieties is proved in [36, Theorem 3.23], but assumptions are made about the base schemes (see the conditions immediately preceding equation 3.8) that do not apply to general deformation problems, including lifting (for example, lifting from $\mathbf{Z} / p \mathbf{Z}$ to $\mathbf{Z} / p^{2} \mathbf{Z}$ ). There is a derived version of the statement in [33, Section 3], but [ibid.] works over $\mathbf{C}$ rather than a general base. There is another derived version at [11] that also assumes the base has characteristic 0. Finally, there is a similar statement at the end of [22], which works over general Noetherian bases, but there are flatness assumptions that do not hold in the present context (or, more generally, in the context of infinitesimal deformation problems where the base is not a field). We will not attempt to give a proof here, so we will refer to this statement as a conjecture rather than a lemma. A write-up that works in sufficient generality will appear in [27].

Lemma 3.1.6. Suppose $\gamma:(Z, \alpha) \rightarrow(X, \beta)$ is an untwisted finite étale morphism of smooth proper twisted schemes of relative dimension 2 over $A$ such that $\omega_{Z / A} \cong \mathcal{O}_{Z}$ and its degree is invertible in $A$. Suppose $Q$ is a perfect complex on $\left(X_{A_{0}}, \beta_{0}\right)$ such that

1. the invertible sheaf $\operatorname{det} Q \in \operatorname{Pic}(Z, \alpha)$ is unobstructed with respect to the extension $\left(Z_{0}, \alpha_{0}\right) \subset(Z, \alpha)$;

2. we have

$$
\mathbf{L} \gamma^{*} Q \cong \bigoplus_{i=1}^{m} Q_{i}
$$

for some $m$ invertible in $A_{0}$ with each $Q_{i}$ a simple perfect complex on $\left(Z_{0}, \alpha_{0}\right)$; 
3. for each $i=2, \ldots, m$ we have

$$
\operatorname{det} Q_{i} \cong \operatorname{det} Q_{1} \otimes \Lambda_{i}
$$

for some $\Lambda_{i} \in \operatorname{Pic}\left(Z_{0}, \alpha_{0}\right)$ that is unobstructed with respect to the extension $\left(Z_{0}, \alpha_{0}\right) \subset(Z, \alpha)$.

Then the complex $Q$ is unobstructed.

Proof. Since the degree $d$ of $\gamma$ is invertible in $A$, the map $\frac{1}{d} \operatorname{tr} \gamma$ is a section of $O_{X} \rightarrow \gamma_{*} \mathcal{O}_{Z}$. Hence, (3.1.2) is injective and by Lemma 3.1.1, it suffices to show that $\mathbf{L} \gamma^{*} Q$ is unobstructed. Further by Conjecture 3.1.3 it suffices to show that $\operatorname{det}\left(Q_{1}\right)$ is unobstructed; note that the simplicity assumption is necessary for (3.1.4) to be injective in the relevant case.

By assumption,

$$
\mathbf{L} \gamma^{*} Q \cong \operatorname{det}\left(Q_{1}\right)^{\otimes m} \otimes \Lambda_{2} \otimes \cdots \otimes \Lambda_{m} .
$$

Since the $\Lambda_{i}$ and $\operatorname{det}(Q)$, and hence $\gamma^{*} \operatorname{det}(Q)$ are unobstructed, we have $m \operatorname{ob}\left(\operatorname{det} Q_{1}\right)=0$. Since $m$ is invertible in $A_{0}$, we conclude that $\operatorname{ob}\left(\operatorname{det} Q_{1}\right)=0$.

The following setting puts us in the situation of either Theorem 1.2 or 1.1.

Setting 3.1.7. Now suppose that $(X, \alpha)$ and $(Y, \beta)$ are twisted schemes that are either smooth proper Enriques or trivially twisted bielliptic surfaces over an Artinian ring $A$ with algebraically closed residue field $k$. Let $n$ be the order of $\omega_{X}$ in $\operatorname{Pic}(X)$. Suppose

$$
\Phi_{P}: D(X, \alpha) \rightarrow D(Y, \beta)
$$

is a Fourier-Mukai equivalence. We assume that the characteristic of $k$ is at least 3 if $X$ is Enriques and at least 5 if $X$ is bielliptic.

Note that in this setting, $\boldsymbol{\mu}_{n}$-gerbes representing $X$ and $Y$ may be chosen as the Brauer group of an Enriques surface has a cardinality of 2 .

Lemma 3.1.8. Suppose we are in the situation described in Setting 3.1.7. Given a section $x \in X(A)$, let $\mathscr{L}_{x}$ denote an invertible $\left.\alpha\right|_{x}$-twisted sheaf. There is a decomposition

$$
\mathbf{L} \pi_{Y}^{*} \Phi_{P}\left(\mathscr{L}_{x}\right) \cong \bigoplus_{i=1}^{n} Q_{i}
$$

in $D\left(\tilde{Y}, \beta_{\tilde{Y}}\right)$ that satisfies the conditions of Lemma 3.1.6.

Proof. Choose a finite closed subgroup $\mathbf{G}\left(=\boldsymbol{\mu}_{n}\right) \subset \mathbf{G}_{m}$ and $\mathbf{G}$-gerbes $\mathscr{X} \rightarrow X$ and $\mathcal{Y} \rightarrow Y$ representing $\alpha$ and $\beta$. Choosing such structures allows us to use the theory of twisted Mukai vectors. Let

$$
\Phi_{\widetilde{P}}: D(\widetilde{\mathscr{X}}) \rightarrow D(\widetilde{\mathscr{Y}})
$$

be the canonical covering equivalence induced by $\Phi_{P}$ as described in Section 2.7. By the commutativity of diagrams (2.7.2), there is an isomorphism

$$
\mathbf{L} \pi_{Y}^{*} \Phi_{P}\left(\mathscr{L}_{x}\right) \cong \Phi_{\widetilde{P}}\left(\mathbf{L} \pi_{X}^{*}\left(\mathscr{L}_{x}\right)\right) .
$$

On the other hand, $\mathbf{L} \pi_{X}^{*}\left(\mathscr{L}_{x}\right)$ can be written as $\mathscr{L}_{x_{1}} \oplus \cdots \oplus \mathscr{L}_{x_{n}}$, where $\left\{x_{1}, \ldots, x_{n}\right\}=\pi_{X}^{-1}(x)$ and $\mathscr{L}_{x_{i}}$ is an invertible $\alpha_{x_{i}}$-twisted sheaf. Note that $\mathscr{L}_{x_{1}}\left|\mathscr{x}_{k}, \ldots, \mathscr{L}_{x_{n}}\right| \mathscr{X}_{k}$ all have the same Mukai vector. It follows from Lemma 2.4.3

$$
\Phi_{\widetilde{P}_{k}}\left(\left.\mathscr{L}_{x_{1}}\right|_{\widetilde{x}_{k}}\right), \ldots, \Phi_{\widetilde{P}_{k}}\left(\left.\mathscr{L}_{x_{n}}\right|_{\widetilde{X}_{k}}\right) \in D\left(\widetilde{\mathscr{Y}}_{k}\right)
$$


have the same Mukai vectors (where $\widetilde{P}_{k}$ denotes the derived restriction of $\widetilde{P}$ to $\widetilde{\mathscr{X}}_{k} \times \widetilde{\mathscr{Y}}_{k}$ ). In particular, the determinants of the complexes $\Phi_{\widetilde{P}_{k}}\left(\left.\mathscr{L}_{x_{i}}\right|_{\widetilde{x}_{k}}\right)$ are equal in $\operatorname{Pic}\left(\mathscr{Y}_{k}\right) \otimes \mathbf{Q}$. It follows that for every $i$ we have that the invertible sheaf

$$
\Lambda_{i}:=\operatorname{det}\left(\Phi_{\widetilde{P}}\left(\mathscr{L}_{x_{i}}\right)\right) \otimes \operatorname{det}\left(\Phi_{\widetilde{P}}\left(\mathscr{L}_{x_{1}}\right)\right)^{\vee}
$$

defines a section of $\operatorname{Pic}_{\tilde{y} / A}^{0}$ over $A_{0}$.

Since $Y$ is either Enriques or bielliptic (so that $\widetilde{Y}$ is either $\mathrm{K} 3$ or abelian), the scheme $\operatorname{Pic}_{\tilde{Y} / A}^{0}$ is smooth over $A$. The hypothesis of Lemma 3.1.6 is thus satisfied.

\subsection{Moduli of perfect complexes of twisted sheaves}

In this section we fix a smooth projective family $\pi:(Y, \alpha) \rightarrow S$ of twisted schemes. We will write $\operatorname{Perf}_{(Y, \alpha) / S}$ for the stack of simple universally gluable relatively perfect complexes of $\alpha$-twisted sheaves, generalizing the notions of [23]; see also [32, §3] for a discussion of this stack. The following proposition summarizes the main properties of $\operatorname{Perf}_{(Y, \alpha) / S}$.

Proposition 3.2.1. Given $(Y, \alpha) \rightarrow S, \mathscr{A}$, and $\mathcal{Y}$ as above, the following hold.

1. The stack $\operatorname{Perf}_{(Y, \alpha) / S} \rightarrow S$ is an Artin stack locally of finite presentation with inertia stack $\mathbf{G}_{m}$. (In particular, it is a $\mathbf{G}_{m}$-gerbe over an algebraic space locally of finite presentation.)

2. Given a deformation situation $A^{\prime} \rightarrow A \rightarrow A_{0}$ with kernel I (in the notation of Artin [2]), the natural obstruction theory for an object $P \in \operatorname{Per}_{(Y, \alpha) / S}(A)$ takes values in $\operatorname{Ext}_{\mathscr{Y}_{A}}^{2}(P, P \stackrel{\mathrm{L}}{\otimes} I)$. The tangent theory takes values in $\operatorname{Ext}_{\mathscr{Y}_{A}}^{1}(P, P \stackrel{\mathbf{L}}{\otimes} I)$.

3. The determinant defines a morphism

$$
\delta: \operatorname{Perf}_{(Y, \alpha) / S} \rightarrow \operatorname{Pic}_{(Y, \alpha) / S}
$$

of algebraic stacks.

Proof. There are several proofs of these statements. A low-technology version is to realize perfect complexes of twisted sheaves as complexes of $\mathscr{A}$-modules for an Azumaya algebra $\mathscr{A}$ with Brauer class $\alpha$, and note that the proofs of [23] carry over mutatis mutandis for $\mathscr{A}$-modules. A higher-tech version is to import the derived techniques of Toën-Vaquié [38], as described in [32]. We will not discuss the details here.

Remark 3.2.2. In the twisted case, we also have the following. Proofs are identical to the proofs in Section 3.1 and Section 4 of [26], mutatis mutandis. Given a pair of $\mathbf{G}_{m}$-gerbes $\mathscr{X} \rightarrow X$ and $\mathscr{Y} \rightarrow Y$ flat and of finite presentation over a base $B$, a Fourier-Mukai kernel $P \in D^{(-1,1)}(\mathscr{X} \times \mathscr{Y})$ induces a morphism

$$
\kappa_{P}: \mathscr{X} \rightarrow \text { Perf }_{y / B}
$$

Proposition 3.2.3. Suppose $\mathscr{X} \rightarrow X$ and $\mathscr{Y} \rightarrow Y$ are $\mathbf{G}_{m}$-gerbes representing smooth twisted (relative) varieties over a base scheme B. Given a Fourier-Mukai equivalence

$$
\Phi_{P}: D^{(1)}(\mathscr{X}) \rightarrow D^{(1)}(\mathscr{Y})
$$

the induced morphism

$$
\kappa_{P}: \mathscr{X} \rightarrow \text { Perf }_{y / B}
$$

is an open immersion. 
Proof. See Proposition 4.4 of [26]. Note that in loc. cit. the morphism considered is the map from $X$ to the sheafification of $\mathscr{P e r}_{\mathcal{y}}$, and the base is assumed to be a field. The proposition claimed here follows from that result (readily modified for twisted sheaves) by Nakayama's lemma combined with the fact that the induced morphism of inertia

$$
\mathbf{G}_{m, \mathscr{X}} \rightarrow \kappa^{*} \mathbf{G}_{m, \mathscr{P} \operatorname{erf} y / B}
$$

is an isomorphism because the functor $\Phi_{P}$ is linear.

Corollary 3.2.4. Suppose $A \rightarrow A_{0}$ is a square-zero extension of rings and $\mathscr{X} \rightarrow X$ and $\mathcal{Y} \rightarrow Y$ are $\mathbf{G}_{m^{-}}$ gerbes representing smooth twisted schemes over $A$. Write $\mathscr{X}_{0} \rightarrow X_{0}$ and $\mathscr{Y}_{0} \rightarrow Y_{0}$ for the restrictions to $A_{0}$. Given a relative Fourier-Mukai kernel

$$
P_{0} \in D^{(-1,1)}\left(\mathscr{X}_{0} \times \operatorname{Spec} A_{0} \mathscr{Y}_{0}\right),
$$

there is at most one object

$$
P \in D^{(-1,1)}(\mathscr{X} \times \operatorname{Spec} A \mathscr{Y})
$$

up to quasi-isomorphism such that $\mathbf{L} i^{*} P \cong P_{0}$, where $i: \mathscr{X}_{0} \times \mathscr{Y}_{0} \rightarrow \mathscr{X} \times \mathscr{Y}$ is the canonical inclusion.

Proof. Since $\kappa_{P_{0}}$ is an open immersion, it has at most one extension to an open immersion $\mathscr{X} \rightarrow$ Perf $_{y / A}$, up to 2 -isomorphism.

\subsection{An isomorphism of deformation functors}

Suppose $k$ is algebraically closed of characteristic at least 3 (resp. 5) and

$$
\Phi_{P}: D^{(1)}(X, \alpha) \rightarrow D^{(1)}(Y, \beta)
$$

is a Fourier-Mukai equivalence.

Lemma 3.3.1. If $X$ is Enriques (resp. bielliptic) then $Y$ is Enriques (resp. bielliptic).

Proof. By Lemma 2.3.4, $X$ and $Y$ have the same dimension and $\omega_{X}$ and $\omega_{Y}$ have the same order. Proposition 2.4.5, together with Poincaré duality, implies that derived equivalent surfaces have the same $\ell$-adic Betti numbers. We conclude that $Y$ is Enriques (resp. bielliptic) using Bombieri-Mumford classification of surfaces in positive characteristic [3].

Assumption 3.3.2. We assume in the rest of this section that $(X, \alpha)$ and $(Y, \beta)$ are twisted Enriques (resp. bielliptic) surfaces over an algebraically closed field $k$ of characteristic at least 3 (resp. at least 5). We also fix $\mathbf{G}_{m}$-gerbes $\mathscr{X} \rightarrow X$ and $\mathcal{Y} \rightarrow Y$ representing $\alpha$ and $\beta$. We fix a Fourier-Mukai equivalence

$$
\Phi_{P}: D^{(1)}(\mathscr{X}) \rightarrow D^{(1)}(\mathcal{Y}) .
$$

Let $A \rightarrow A_{0}$ be a map of Artinian augmented $W(k)$-algebras. Suppose $\mathscr{X}_{0} \rightarrow X_{0}$ is a $\mathbf{G}_{m}$-gerbe representing a point $\left[\mathscr{X}_{0}\right] \in \operatorname{Def}_{(X, \alpha)}\left(A_{0}\right)$ and $\mathscr{Y}_{A} \rightarrow Y_{A}$ is a $\mathbf{G}_{m}$-gerbe representing a point $\left[\mathscr{Y}_{A}\right] \in$ $\operatorname{Def}_{(Y, \beta)}(A)$. Write $\mathscr{Y}_{0} \rightarrow Y_{0}$ for the restriction of $\mathscr{Y}_{A} \rightarrow Y_{A}$ to $A_{0}$.

Suppose

$$
\Phi_{P_{0}}: D^{(1)}\left(\mathscr{X}_{0}\right) \rightarrow D^{(1)}\left(\mathscr{Y}_{0}\right)
$$

is a relative Fourier-Mukai equivalence. There is an associated open immersion

$$
\kappa: \mathscr{X}_{A_{0}} \hookrightarrow \operatorname{Perf}_{y_{0} / A_{0}}
$$

of Artin stacks. 
Proposition 3.3.3. The morphism $\kappa$ has image contained in the smooth locus of the structure morphism

$$
\operatorname{Perf}_{y_{A} / A} \rightarrow \operatorname{Spec} A .
$$

Proof. Since the smooth locus is open, it suffices to prove the result under the assumption that $A_{0}=k$, and then it is enough to prove that for any $x \in X(k)$ and any invertible twisted sheaf $\mathscr{L}_{x}$ supported on $x$, the image complex $\Phi\left(\mathscr{L}_{x}\right) \in D(Y, \beta)$ lies in the smooth locus of $\mathscr{P e r}_{\mathscr{Y}_{A} / A}$. This follows immediately from Lemma 3.1.8.

Theorem 3.3.1. Under Assumption 3.3.2, there is an isomorphism of formal deformation functors

$$
\rho: \operatorname{Def}_{(Y, \beta)} \rightarrow \operatorname{Def}_{(X, \alpha)}
$$

with the following property. Given a point

$$
\left[\mathscr{Y}_{A} \rightarrow Y_{A}\right] \in \operatorname{Def}_{\mathscr{Y}}(A)
$$

with image

$$
\left[\mathscr{X}_{A} \rightarrow X_{A}\right]=\rho\left(\left[\mathscr{Y}_{A} \rightarrow Y_{A}\right]\right) \in \operatorname{Def}_{\mathscr{X}}(A)
$$

there is a complex

$$
P \in D^{(-1,1)}(\mathscr{X} \times \operatorname{Spec} A \mathcal{Y}),
$$

unique up to quasi-isomorphism, such that $\mathbf{L} i^{*} P_{A} \cong P$, where

$$
i: \mathscr{X} \times_{\operatorname{Spec} k} \mathscr{Y} \rightarrow \mathscr{X}_{A} \times_{\operatorname{Spec} A} \mathscr{Y}_{A}
$$

is the natural closed immersion.

Proof. Given $\mathscr{Y}_{A} \rightarrow Y_{A}$, Proposition 3.2.3 and Proposition 3.3.3 imply that $\kappa_{P}$ identifies $\mathscr{X}$ with an open substack of $\mathscr{P e r}_{Y / k}$ that lies in the smooth locus of $\mathscr{P e r}_{\mathscr{Y}_{A} / A}$. Since open substacks of the smooth locus lift uniquely, we get an induced deformation $\mathscr{X}_{A} \rightarrow X_{A}$ that is a $\mathbf{G}_{m}$-gerbe, giving a point of $\operatorname{Def}_{X}(A)$. Restricting the universal complex gives the desired kernel $P_{A}$. (That $P_{A}$ also gives an equivalence follows from Nakayama's Lemma as in [26, Theorem 6.1].)

By Proposition 2.5.1, for any strictly Henselian local ring $R$ with residue field $k$ and any lift $Y_{R} / R$ (resp. $X_{R} / R$ ), restriction defines an isomorphism $\operatorname{Br}\left(Y_{R}\right) \rightarrow \operatorname{Br}(Y)$ (resp. $\operatorname{Br}\left(X_{R}\right) \rightarrow \operatorname{Br}(X)$ ). Thus, for any such lift, there is a canonical way to propogate any twisting class. Given $\alpha \in \operatorname{Br}(Y)$ and a lift $Y_{R} / R$, we will write $\alpha_{R}$ for the canonical lifting of $\alpha$.

Theorem 3.3.2. Let $X, Y$, and $P$ be as in Assumption 3.3.2. For any complete local Noetherian ring $R$ with residue field $k$ and any lift $Y_{R} \rightarrow \operatorname{Spec} R$ of $Y / k$, there is a lift $X_{R} \rightarrow \operatorname{Spec} R$ such that $\Phi_{P}$ lifts to a relative Fourier-Mukai equivalence

$$
\Phi_{P_{R}}: D^{(1)}\left(X_{R}, \alpha_{R}\right) \rightarrow D^{(1)}\left(Y_{R}, \beta_{R}\right)
$$

Proof. Given a deformation $Y_{R}$ of $Y$, from Theorem 3.3.1 we get an induced formal deformation $\mathfrak{X}_{R} \in$ $\operatorname{Def}_{X}(\operatorname{Spf} R)$ and

$$
\mathfrak{P}_{R} \in D^{(1)}\left(\mathfrak{X}_{R} \times_{\text {Spf } R} \widehat{Y}_{R}, \alpha_{R}^{-1} \otimes \beta_{R}\right) .
$$

Since $\mathrm{H}^{2}(X, \mathcal{O})=0$, any ample invertible sheaf on $X$ lifts to $\mathfrak{X}_{R}$, so we can algebraize $\mathfrak{X}_{R}$ to the completion of a relative Enriques surface $X_{R}$ that carries the canonical Brauer class $\alpha_{R}$ algebraizing the 
formal lift. The Grothendieck Existence Theorem for perfect complexes [23, Proposition 3.6.1] algebraizes $\mathfrak{P}_{R}$ to a complex

$$
P_{R} \in D\left(X_{R} \times_{\operatorname{Spec} R} Y_{R}, \alpha_{R}^{-1} \otimes \beta_{R}\right) .
$$

As in [26, Proof of Theorem 6.1], Nakayama's Lemma then implies that $P_{R}$ is a relative twisted FourierMukai equivalence, as desired.

Remark 3.3.4. In using the Grothendieck Existence Theorem for perfect complexes in the above proof, we are observing that the methods of [23] may be extended to the twisted case. The reader may find it interesting to note that, following the appearance of this paper, Ben Lim has proved a significant strenghtening of this result to perfect complexes on algebraic stacks [28], using methods distinct from [23].

\section{Proof of Theorem 1.1 and Theorem 1.2}

Let $F: D^{(1)}(X, \alpha) \rightarrow D^{(1)}(Y, \beta)$ be an equivalence as in the statement of Theorem 1.1 or 1.2. By [8, Theorem 1.1], there is a kernel $P \in D\left(X \times Y, \alpha^{-1} \otimes \beta\right)$ so that $F$ is naturally isomorphic to the Fourier-Mukai equivalence $\Phi_{P}$. Enriques (resp. bielliptic) surfaces of characteristic at least 3 (resp. 5) can be lifted to a finite extension of the Witt vectors; see [21, Proposition 6.1.1] and Partsch [31]. By Theorem 3.3.2, given any lift $Y_{R}$ of $Y$ over a finite flat $W(k)$-algebra $R$ there are induced deformations $X_{R}$ and $P_{R} \in D\left(X_{R} \times_{\operatorname{Spec} R} Y_{R}, \alpha_{R}^{-1} \otimes \beta_{R}\right)$ giving a relative Fourier-Mukai equivalence.

By [23, Proposition 3.6.1], for any ring homomorphism $R \rightarrow S$, the base change $P_{S}$ induces an equivalence

$$
D^{(1)}\left(X_{S}, \alpha_{S}\right) \rightarrow D^{(1)}\left(Y_{S}, \beta_{S}\right)
$$

In particular we may choose an embedding $\kappa(R) \rightarrow \mathbf{C}$, yielding a Fourier-Mukai equivalence

$$
\Phi_{P_{\mathbf{C}}}: D\left(X_{\mathbf{C}}, \alpha_{\mathbf{C}}\right) \simeq D\left(Y_{\mathbf{C}}, \beta_{\mathbf{C}}\right) .
$$

If $\alpha$ and $\beta$ are 0 then [6, Proposition 6.1, 6.2] implies that $X_{\mathbf{C}}$ and $Y_{\mathbf{C}}$ are isomorphic. If $X$ and $Y$ are Enriques and $\alpha$ and $\beta$ are arbitrary, then [1] ensures that $\left(X_{\mathbf{C}}, \alpha_{\mathbf{C}}\right)$ and $\left(Y_{\mathbf{C}}, \beta_{\mathbf{C}}\right)$ are isomorphic.

Spreading out, we find a finite extension $K^{\prime}$ of $\kappa(R)$ such that there is an isomorphism

$$
f:\left(X_{K^{\prime}}, \alpha_{K^{\prime}}\right) \rightarrow\left(Y_{K^{\prime}}, \beta_{K^{\prime}}\right)
$$

over $K^{\prime}$. The normalization $R^{\prime}$ of $R$ in $K^{\prime}$ is a complete DVR with residue field $k$ and fraction field $K^{\prime}$. Since invertible sheaves are unobstructed, this isomorphism preserves relative polarizations over $R^{\prime}$. Hence, we may use [29, Theorem 2] to conclude that the isomorphism $f$ induces an isomorphism $f: X_{R^{\prime}} \rightarrow Y_{R^{\prime}}$ of the underlying schemes. By Proposition 2.5.1, we see that $f^{*} \beta_{R}=\alpha_{R}$. Specializing to $k$ gives the desired result.

\section{References}

[1] N. Addington and A. Wray. Twisted Fourier-Mukai partners of Enriques surfaces. arxiv: 1803.03250.

[2] M. Artin. Versal deformations and algebraic stacks. Invent. Math., 27:165-189, 1974. 
[3] E. Bombieri and D. Mumford. Enriques' classification of surfaces in char. p. II. pages 23-42, 1977.

[4] D. Bragg and M. Leiblich. Twistor spaces for supersingular K3 surfaces. arXiv: 1804.07282, 2019.

[5] A. Bridgeland, T.and Maciocia. Fourier-Mukai transforms for quotient varieties. Journal of Geometry and Physics, 2017.

[6] T. Bridgeland and A. Maciocia. Complex surfaces with equivalent derived categories. Math. Z., 236(4):677-697, 2001.

[7] J. Bryan, C. Cadman, and B. Young. The orbifold topological vertex. Adv. Math., 229:531-595, 2012.

[8] A. Canonaco and P. Stellari. Twisted Fourier-Mukai functors. Adv. Math., 212:484-503, 2007.

[9] A. Căldăraru. Derived categories of twisted sheaves on Calabi-Yau manifolds. PhD thesis, Cornell University, 2000.

[10] J. de Jong. A result of Gabber. https://www.math.columbia.edu/ dejong/papers/2-gabber.pdf.

[11] D. Gaitsgory and N. Rozenblyum. A study in derived algebraic geometry. Vol. II. Deformations, Lie theory and formal geometry, volume 221 of Mathematical Surveys and Monographs. American Mathematical Society, Providence, RI, 2017.

[12] H. Gillet. Intersection theory on algebraic stacks and Q-varieties. Journal of Pure and Applied Algebra, 34(2):193-240, 1984.

[13] J. Giraud. Cohomologie non abéllienne., volume 179 of Grundlehren der mathematischen Wissenschaften. 1971.

[14] A. Grothendieck. Éléments de géométrie algébrique: III. Étude cohomologique des faisceaux cohérents. II. Inst. Hautes Études Sci. Publ. Math., (17):91, 1963.

[15] Alexander Grothendieck. Le groupe de Brauer. I. Algèbres d'Azumaya et interprétations diverses. In Dix Exposés sur la Cohomologie des Schémas, pages 46-66. North-Holland, Amsterdam; Masson, Paris, 1968.

[16] K. Honigs. Derived equivalent surfaces and abelian varieties, and their zeta functions. Proc. Amer. Math. Soc., 143(10):4161-4166, 2015.

[17] D. Huybrechts. Fourier-Mukai transforms in algebraic geometry. Oxford Mathematical Monographs. The Clarendon Press Oxford University Press, Oxford, 2006.

[18] D. Huybrechts. Motives of isogenous K3 surfaces. arXiv:1705.04063, 2018.

[19] D. Huybrechts and P. Stellari. Equivalences of twisted K3 surfaces. Math. Ann., 332(4):901-936, 2005.

[20] Luc Illusie. Complexe cotangent et déformations, volume 239 of Lecture notes in mathematics (SpringerVerlag). 1971.

[21] Luc Illusie. Grothendieck's existence theorem in formal geometry. In Fundamental algebraic geometry, volume 123 of Math. Surveys Monogr., pages 179-233. Amer. Math. Soc., Providence, RI, 2005. With a letter of Jean-Pierre Serre. 
[22] F. Langholf. Atiyah classes with values in the truncated cotangent complex. Math. Nachr., 286(13):1305-1325, 2013.

[23] M. Lieblich. Moduli of complexes on a proper morphism. J. Algebraic Geom., 15(1):175-206, 2006.

[24] M. Lieblich. Moduli of twisted sheaves. Duke Math.J., 138(1):23-118, 2007.

[25] M. Lieblich. Twisted sheaves and the period-index problem. Compos. Math., 144(1):1-31, 2008.

[26] M. Lieblich and M. Olsson. Fourier-Mukai partners of K3 surfaces in positive characteristic. Ann. Sci. Éc. Norm. Supér. (4), 48(5):1001-1033, 2015.

[27] Max Lieblich and Martin Olsson. Deformations of perfect complexes and trace maps. in preparation.

[28] D. B. Lim. GrothendieckâĂŹs existence theorem for relatively perfect complexes on algebraic stacks. arxiv: 1907.05025 . preprint.

[29] T. Matsusaka and D. Mumford. Two fundamental theorems on deformations of polarized varieties. American Journal of Mathematics, pages 668-684, 1964.

[30] M. Olsson. Algebraic Spaces and Stacks, volume 62 of Colloquium Publications. Amer. Math. Soc., 2016.

[31] H. Partsch. Deformations of elliptic fibre bundles in positive characteristic. Nagoya Math. J., 211:79108, 2013.

[32] E. Reinecke. Autoequivalences of twisted K3 surfaces. arXiv:1711.00846, 2017.

[33] T. Schürg, B. Toën, and G. Vezzosi. Derived algebraic geometry, determinants of perfect complexes, and applications to obstruction theories for maps and complexes. J. Reine Angew. Math., 702:1-40, 2015.

[34] N. Spaltenstein. Resolutions of unbounded complexes. Compositio Math., 65(2):121-154, 1988.

[35] The Stacks Project Authors. Stacks Project. http://stacks.math.columbia.edu, 2018.

[36] R. P. Thomas. A holomorphic Casson invariant for Calabi-Yau 3-folds, and bundles on $k 3$ fibrations. J. Differential Geom., 54(2):367-438, 2000.

[37] B. Toën. Théorèmes de Riemann-Roch pour les champs de Deligne-Mumford. K-Theory, 18:33-76, 1999.

[38] B. Toën and M. Vaquié. Moduli of objects in dg-categories. Ann. Sci. École Norm. Sup. (4), 40(3):387444, 2007.

[39] K. Yoshioka. Moduli spaces of twisted sheaves on a projective variety. In Moduli spaces and arithmetic geometry, volume 45 of Adv. Stud. Pure Math., pages 1-30. Math. Soc. Japan, Tokyo, 2006. 